\title{
Imaging in blunt abdominal trauma; a review in current literature
}

\author{
Mojahed Hadi Ali Rudainee ${ }^{1}$, Tahani Saleh A. Alsaery ${ }^{2}$, Ghaida Wael Khayat ${ }^{3}$, Majed \\ mohammed fahed Alharbi ${ }^{4}$, Mazen Hassan Sadoun Alaslani ${ }^{5}$, Sarah Mohammed Mahrous ${ }^{4}$, \\ Muhammad Al Ahmad ${ }^{6}$, Yara Mofarih Assiri ${ }^{7}$, Arwa Musaad Alsubhi ${ }^{4}$, Nader Awad \\ Alanazi $^{8}$
}

1-Aseer Central Hospital , 2- Resident at KFAFH , 3-Ibn Sina National College for Medical Studies , 4- Taibah University , 5- Gp of Comprehensive clinics of minstory of interior-Riyadh , 6- Oyun City Hospital , 7- King khalid university , 8- Imam Muhammad Ibn Saud Islamic University

\begin{abstract}
Introduction: The common imaging modalities used for the diagnosis of pelvic and abdominal trauma range from ultrasonography, X-ray, computed tomography, and others. In each different kind of abdominal condition, a different modality is preferred depending on the nature of condition, the patient, and the hospital facility where the management is provided. Some conditions require more than one source of imaging. Aim of the work: In this study, our aim was to discuss various abdominal and pelvic pathologies separately to explore the preferred type of imaging modality. Methodology: we conducted this review using a comprehensive search of MEDLINE, PubMed and EMBASE from January 1994 to March 2017. The following search terms were used: ultrasound versus CT, abdominal radiology, acute abdomen imaging, pelvic pain diagnosis. Conclusion: Blunt abdominal traumas are very common with many causes including motor vehicle accidents, bicycle accidents, and falls. Appropriate and early management of blunt abdominal trauma is essential to prevent the occurrence of significant long-term morbidities and high mortality rates. Due to nonspecific clinical manifestation, establishing a proper diagnosis mainly depends on radiologic modalities.
\end{abstract}

Keywords: abdominal trauma, ultrasound, computed tomography, acute abdomen, gynecologic emergency, abdominal imaging.

\section{Introduction:}

When suffering from a blunt abdominal trauma, the liver is the most common organ to be injured followed by the spleen and then the mesenteries and bowels ${ }^{(1)}$. Radiologists who are not sufficiently experienced can sometime miss the presence of mesenteries or bowels injuries. The main reason behind this is that these injuries are not the most common, thus radiologists do not think of them immediately. In fact, the incidence of a bowel injury in the bowels is reported to be about $3 \%$ in cases of blunt abdominal trauma. This incidence increases in cases of the presence of other visceral injuries and can reach $34 \%$ when there are injuries in more than three organs following a blunt trauma ${ }^{(2)}$. Moreover, signs and symptoms associated with them are not always clear and specific, leading to relatively high rates of missing the diagnosis. Unfortunately, this delay in diagnosis, even for few hours, can lead to the development of significant long-term morbidities, and high rates of mortality. Complications can include peritonitis and sepsis ${ }^{(3)}$. Therefore, mesenteries and bowels injuries are considered to be challenging for radiologists. Findings of mesenteries and bowels injuries are not always clear on a CT scan, making it difficult to make the decision of operating or not. Therefore, it is essential to obtain sufficient experience that allows radiologists to be able to distinguish between cases and make the right decisions.

All this make the role of abdominal imaging extremely essential in the work up of blunt abdominal trauma. Radiologists, in these cases, are responsible to make the final decision regarding diagnosis, management, and possible treatment ${ }^{(4)}$.

\section{Methodology:}

We did a systematic search for blunt abdominal trauma and imaging using PubMed search engine (http://www.ncbi.nlm.nih.gov/) and Google Scholar search engine (https://scholar.google.com). Our search looked for radiology modalities that are used in cases of blunt abdominal trauma. All relevant studies were retrieved and discussed. We only included full articles. The following 
search terms were used: ultrasound versus CT, acute abdomen imaging, abdominal radiology, pelvic pain diagnosis. The study was approved by the ethical board of King Abdulaziz University.

\section{Anatomical and physiological considerations:}

When suffering from a blunt abdominal trauma that injures the bowels, the most common site to be injured is the small intestines, which can be found to be injured in up to $70 \%$ of cases ${ }^{(5)}$. Within the small intestines, most injuries occur in the proximal part of the jejunum, the distal part of the ileum, or bowel segments that are near to adhesions, making them highly exposed to damage ${ }^{(6)}$.

On the other hand, the large intestines are rarely injured from blunt abdominal traumas. In fact, only $0.5 \%$ of patients who suffered a blunt abdominal trauma can be found to have a large intestine injury, with most of these cases being only partial ${ }^{(7)}$.

Being retroperitoneal, and near to the thoracic spine, the duodenum is relatively less injured than other bowel organs when suffering a blunt abdominal trauma ${ }^{(6)}$. The primary mechanism of duodenal injury following a blunt abdominal trauma is rapid deceleration which will lead to the development of significant tearing near the junction of the retroperitoneal and the intraperitoneal parts (the third and fourth parts of the duodenum) ${ }^{(1)}$.

Motor vehicle accidents represent the most common cause of blunt abdominal trauma leading to visceral injuries and constitute up to $85 \%$ of cases. Other less common causes of blunt abdominal trauma include aggressions and falls ${ }^{(7)}$. The use of seat belts has been associated with a significant increase in the incidence of bowels injuries in motor vehicle accidents. The mechanisms behind this is suggested to be due to the compression of bowels loops, which will lead to the creation of a closed viscus. This will in turn elevate the pressure within the lumens leading to a bursting injury. Therefore, the 'seat belt mark' sign has been suggested to be a specific finding for predicting the presence of mesenteries or bowels injuries ${ }^{(8)}$.

In children, the incidence of bowel injuries following a blunt abdominal trauma is significantly higher than adults. The possible reason behind this is the immature abdominal wall muscles. Moreover, in this age group, injuries from following bicycles' accidents are a common ${ }^{(9)}$.

In general, mechanisms that will lead to the development of organs injury following a blunt abdominal trauma are counted here ${ }^{(7)}$ :

- Crushing of the gastrointestinal tract between the abdominal wall and the vertebrae, which is caused by direct force. An example of this is injuries with seat belt or bicycles.

- Shearing forces between mobile parts and fixed parts of the organ, which are caused by fast deceleration.

- The sudden elevation of pressure in the lumen of organs, which leads to bursting of loops.

\section{Clinical presentation:}

Clinically, only half (or even less) of patients who had a bowel or mesenteric injury following an abdominal trauma, showed signs and symptoms of peritonitis. In most cases, classic peritonitis signs were not obvious early. Sometimes, the neurological impairments that were caused by concomitant injuries can mask the guarding ${ }^{(10)}$. Therefore, determining the need of laparotomy, based only on clinical assessment, had been estimated to be associated with up to $40 \%$ negative rates ${ }^{(3)}$.

Until now, no laboratory findings had been found to have sufficient specificity for detecting visceral injuries following a blunt abdominal trauma. The use of diagnostic peritoneal lavage had been suggested to have a relatively high sensitivity in diagnosing such injuries. However, it had a significantly low specificity, and was associated with high rates of false negatives ${ }^{(3)}$. Therefore, imaging modalities are the most important measures in diagnosing and managing injuries following blunt abdominal traumas ${ }^{(7)}$.

\section{Conventional radiography $(\mathrm{CR})$ :}

The use of conventional radiograph ( $\mathrm{x}$ ray) in the detection of visceral injury following a blunt abdominal trauma is limited to the detection of extraluminal free air ${ }^{(11)}$.

\section{Ultrasonography (US):}

Currently, the Focused Assessment with Sonography for Trauma 'FAST' is the universally accepted and validated modality for the initial assessment of any patient who is suspected to have visceral injury following a blunt abdominal trauma. Using Focused 
Assessment with Sonography for Trauma, even minimal bowel injuries or mesenteries injuries that lead to little peritoneal fluid leakage could be identified. However, despite being highly sensitive, this finding is not specific enough and can lead to high false negative rates, especially in females in childbearing age, or with the presence of coexisting morbidities like liver failure ${ }^{(12)}$.

Moreover, focal-segmental injuries or abnormal echogenicity following a blunt abdominal are not commonly detected with FAST technique. The main reason behind this is that these injuries commonly occur in retroperitoneal structures, which are relatively deep and hard to be recognized with ultrasound ${ }^{(12)}$.

\section{Computed tomography:}

The use of multi-detector computed tomography is considered today to be the most important modality when diagnosing visceral injuries following a blunt abdominal trauma in both stable and unstable patients. Multidetector computed tomography has been found to have ranging sensitivity and specificity that can be as high as $95 \%$ and $100 \%$, respectively. These high sensitivity and specificity are a result of several factors including high resolutions of contrast and the ability to obtain much information from both hollow and parenchymatous visceral organs, within only few seconds ${ }^{(13)}$. Moreover, the accuracy of multi-detector computed tomography in the detection of visceral injuries following a blunt abdominal trauma could be potentially increased with the acquisition of faster and thinner sections ${ }^{(14)}$. Therefore, despite their availability, conventional radiography and ultrasound are still limited in diagnosing visceral injuries when these techniques are compared to that of multi-detector computed tomography.

When dealing with a case of suspected visceral injury following a blunt abdominal trauma, multi-detector computed tomography should be done following the 'high-resolution' protocol, in which the thickness of the slice is $1 \mathrm{~mm}$ and is later completed using multiplanar reconstructions. Performing a CT scan before injecting contrast medium is important when suspecting a visceral injury for many reasons including ${ }^{(7)}$ :

- This will aid in the identification of accumulating free air when a perforation is present.
- This will improve the assessment of any fluid collection in the abdomen or in bowels walls. Moreover, this will help differentiate between a normal lower density collection, and a hematoma caused by the trauma.

Following the injection of iodinated contrast (about 120 to $150 \mathrm{ml}$, in a rate that is more than $3 \mathrm{ml}$ per second), it is recommended to do an arterial and venous biphasic assessment to detect possible bleeding and perfusion defects in the bowel loops. About five minutes following the injection of the contrast material, a late phase acquisition can be helpful to rule out the presence of an active bleeding. On the other hand, the use of oral contrast material in the diagnosis of suspected visceral injury following a blunt abdominal trauma has been an area of debate. Most current guidelines are against the use of oral contrast in these cases. Their rationale behind this is the waste of time without the presence of a clear benefit. Moreover, this oral contrast may distribute to other organs and lead to misdiagnoses of cases, especially when this contrast is spilled of the bladder when there is a traumatic rupture of the bladder ${ }^{(7)}$.

\section{Features and CT classification of traumatic visceral injuries following a blunt abdominal trauma:}

Several factors contribute to the end effect of the injury on the viscera and bowels. These factors include the type and severity of the trauma, the anatomical characteristics of the organ on which the force was applied, luminal distension and the degree of it, along with other possible factors. When dealing with cases of intestinal injuries, these cases are usually classified into 'major' and 'minor' (15). An intestinal injury is only considered 'major' when it is a full thickness injury that creates a continuity between the peritoneum and the intestinal lumen leading to spilling of intestinal contents into the peritoneum. These cases are considered major due to the high risk of developing chemical peritonitis, and therefore require immediate and proper management and treatment to prevent late complications ${ }^{(5)}$.

On the other hand, an intestinal injury that is limited to a part of the intestinal wall is considered 'minor'. Examples of these include parietal contusions, incomplete bowels wall tears, and intramural hematomas. These cases are considered minor due to the rarity of 
secondary perforation caused by them. Therefore, can be sufficiently managed without surgery ${ }^{(16)}$.

When dealing with both minor and major intestinal injuries, non-specific and specific CT findings can be detected, and together will help establishing the diagnosis. Specific signs are many and include visualizing the interruption of the intestinal wall directly, the presence of free enteric contents (like feces or oral contrast) in the abdominal cavity, and the detection of parietal hematoma. On the other hand, collection of air out of the lumen, intestinal wall thickening, mesentery infiltration, the presence of fluid within the peritoneum, and the abnormal enhancement are all considered not specific CT signs for detecting the presence of an intestinal injury (16).

Intestinal wall interruption is considered one of the most specific signs for the detection of an intestinal wall injury, with a specificity that can reach $100 \%$ (7). However, it is associated with an extremely low sensitivity that can be as low as $7 \%$. The reason behind this is that most intestinal lesions are small and do not progress to involve the whole of the intestinal wall ${ }^{(17)}$. The spillage of bowel contents (feces, fluids, ingested food, or oral contrast) into the abdominal cavity is also associated with a very high specificity, but still has a sensitivity that is as low as $12 \%{ }^{(18)}$. Similarly, intramural hematoma can have a very high specificity in detecting intestinal injury following a blunt abdominal trauma, but it has a very low sensitivity due to being very hard to detect. In fact, it cannot be recognized without an extremely careful analysis of cases that have already been diagnosed to have an intestinal injury ${ }^{(19)}$. The presence of an intramural hematoma is generally more common in injuries in the duodenum and rare in injuries in the colon ${ }^{(9)}$. CT in cases of duodenal injury can cause findings of fluid collection to be associated with bowel thickening, making it harder to detect hematoma and differentiate it from perforation. The presence of air collection in the abdominal cavity can help diagnosis of a duodenal perforation in these cases, and conservative management is usually the best approach, as most cases will resolve within less than a month ${ }^{(7)}$. In rare cases, spontaneous resolution does not occur, and the case can complicate into obstruction and/or luminal stenosis ${ }^{(19)}$.

The collection of air in the peritoneum, or the mesenteries is considered to be a highly sensitive sign for bowel injury following a blunt abdominal trauma, despite having a low specificity ${ }^{(20)}$. Even when other specific signs of intestinal injury are absent, the presence of air collection should always lead to further management. Air collection can sometimes be associated with other signs like abnormal parietal enhancement, thickening of the intestines wall, the presence of free fluid in the peritoneum, and the infiltration of the mesenteries. All these signs can make a diagnosis of intestinal injury more likely, and help differentiating it from other causes. However, caution should be applied to differentiate this sign from another similar sign called 'pseudopneumoperitoneum'. Pseudopneumoperitoneum is defined as the entrapment of air between the parietal layer of the peritoneum and the abdominal wall. Pseudopneumoperitoneum can be very similar to true pneumoperitoneum leading to a wrong diagnosis ${ }^{(20)}$.

In many intestinal injury cases following a blunt abdominal trauma, the presence of fluid in the peritoneal cavity can be the first and only sign on CT scanning (21). This is in contrast to other signs like pneumoperitoneum that may not be visible for several hours following the trauma. Therefore, in patients whose CT scan show intraperitoneal fluid only, the best management is a follow up CT scan after six to eight hours ${ }^{(22)}$. Moreover, it is important to distinguish between physiologic and non-physiologic (or pathologic) accumulation of intraperitoneal fluids. Accumulation of free fluid in the peritoneal cavity is considered pathologic when it exceeds $25 \mathrm{ml}$ in adult males, $75 \mathrm{ml}$ in childbearing aged females, or 25 in children. In cases of the absence of any other signs that indicate a solid organ injury, along with the presence of physiologic free fluid in the peritoneum, the suspicion of a possible injury in a hollow viscus should be raised ${ }^{(21,22)}$.

The distribution of free fluid in the peritoneal cavity is helpful in detecting the organ that is injured. For example, when injury occurs in the liver or in the spleen, the free fluid is usually detected in subphrenic spaces and in the pelvis. However, when the injury is in the bowels or the mesenteries, fluid is more 
commonly found in the fold of the mesenteries and loops leading to the formation of a classical polygonal collection. When there is a serosal laceration, fluid can spread in a ' $\mathrm{V}$ Shaped' pattern in the folds of the mesenteries, with the base of the $\mathrm{V}$ is the loop and apex is the root of mesenteries. Finally, when the injury occurs in a retroperitoneal viscus, fluid usually remains localized in to the injury site (16)

Other than the distribution of the intraperitoneal fluid, the density of the fluid is another characteristic that help detect the exact site of the injury. Fluid density is categorized into low- medium-or high- and is determined by comparing it with bile or urine in the gallbladder or urinary bladder, respectively). For example, when there is spillage of enteric contents from the bowels, fluid tends to be of low density. However, collection of fluid with medium density is generally associated with the accumulation of blood, and collection of fluid with high-density is usually associated with spillage of contrast material ${ }^{(7,23)}$. In conclusion, the density of free fluid can help detecting the source of injury but is still not an absolute diagnostic tool.

Intestinal wall thickening is visualized on CT scanning as a disproportionate thickening of intestinal walls that is more than $3 \mathrm{~mm}$. This sign is associated with a low specificity but a relatively high sensitivity in detecting intestinal injury following blunt abdominal trauma, when compared to other signs like enteric contents spillage ${ }^{(7)}$. However, an intestinal loop thickening that is limited only to the declivous are of intestinal loops should not be considered a sign of intestinal injury, as it is mainly caused by bowel contents (24). Moreover, several plausible causes also cause intestinal wall thickening and may lead to false-positive results. These include the presence of another inflammatory or infectious disease, the interruption of venous or arterial supply drainage, and hematoma due to nontraumatic etiologies ${ }^{(24)}$.

\section{Conclusions:}

Blunt abdominal traumas are very common with many causes including motor vehicle accidents, bicycle accidents, falls, along with other possible causes. A blunt abdominal trauma can most commonly cause injuries in the liver, spleen, and intestines. Appropriate and early management of blunt abdominal trauma is essential to prevent the occurrence of significant long-term morbidities and high mortality rates. Due to non-specific clinical manifestation, establishing a proper diagnosis mainly depends on radiologic modalities. A right accurate diagnosis is the first step to assess the need for surgery and to plan proper management and treatment. Liver and spleen injuries can usually be detected easily with ultrasound. However, intestinal and mesenteric injuries are usually more difficult and harder to detect and diagnose. CT scan is considered to be the modality of choice to detect an intestinal injury. In cases of intestinal injuries, CT scan can show specific signs like visualizing the interruption of the intestinal wall directly, the presence of free enteric contents (like feces or oral contrast) in the abdominal cavity, and the detection of parietal hematoma. Other possible signs can be present but are considered non-specific. These include collection of air out of the lumen, intestinal wall thickening, mesentery infiltration, the presence of fluid within the peritoneum and the abnormal enhancement. All specific and non-specific signs should be will studied and taken into consideration when dealing with a blunt abdominal trauma case, especially when there is a high suspicion of visceral injury.

\section{References:}

1. Patlas MN, Alabousi A, Scaglione M, Romano L, Soto JA (2013): Crosssectional imaging of nontraumatic peritoneal and mesenteric emergencies. Can Assoc Radiol J., 64(2): 148-153.

2. Biswas S, Adileh M, Almogy G, Bala M (2014): Abdominal injury patterns in patients with seatbelt signs requiring laparotomy. J Emerg Trauma Shock, 7 (4): 295-300.

3. Malinoski DJ, Patel MS, Yakar DO, Green D, Qureshi F, Inaba K, Brown CV, Salim A (2010): A diagnostic delay of 5 hours increases the risk of death after blunt hollow viscus injury. $\mathrm{J}$ Trauma, 69 (1): 84-87.

4. Walker ML, Akpele I, Spence SD, Henderson $V$ (2012): The role of repeat computed tomography scan in the evaluation of blunt bowel injury. Am Surg., 78 (9): 979-985.

5. Cho HS, Woo JY, Hong HS, Park MH, Ha HI, Yang I, Lee Y, Jung AY, Hwang JY (2013): Multidetector CT findings of bowel transection in blunt 
abdominal trauma. Korean J Radiol., 14 (4): 607- 615.

6. Walker ML (2013): Bowel injury. Minerva Chir., 68 (3): 233-240.

7. Suresh A et al. (2015): Frequency, causes and pattern of abdominal trauma: A 4-year descriptive analysis. J Emerg Trauma Shock, 8(4): 193-198.

8. Borgialli DA, Ellison AM, Ehrlich P, Bonsu B, Menaker J, Wisner DH, Atabaki S, Olsen CS, Sokolove PE, Lillis K, Kuppermann N, Holmes JF (2014): Association between the seat belt sign and intra-abdominal injuries in children with blunt torso trauma in motor vehicle collisions. Acad Emerg Med., 21(11):1240-8

9. Klin B, Efrati Y, Vaiman M, Kozer E, Jeroukhimov I, Abu-Kishk I (2014): Abdominal injuries following bicycle-related blunt abdominal trauma in children. Minerva Pediatr., 68(3):167-72.

10. Joseph DK, Kunac A, Kinler RL, Staff I, Butler KL (2013): Diagnosing blunt hollow viscus injury: is computed tomography the answer? Am J Surg., 205 (4): 414-418.

11. Grassi R, Di Mizio R, Pinto A, Romano L, Rotondo A (2004): Serial plain abdominal film findings in the assessment of acute abdomen: spastic ileus, hypotonic ileus, mechanical ileus and paralytic ileus. Radiol Med., 108: 56-70.

12. Körner M, Krötz MM, Degenhart C, Pfeifer KJ, Reiser MF, Linsenmaier U (2008): Current role of emergency US in patients with major trauma. Radiographics, 28 (1): 225-242.

13. Yu J, Fulcher AS, Turner MA, Cockrell C, Halvorsen RA (2011): Blunt bowel and mesenteric injury: MDCT diagnosis. Abdom Imaging, 36 (1): 50-61.

14. Park MH, Shin BS, Namgung H (2013): Diagnostic performance of 64MDCT for blunt small bowel perforation. Clin Imaging, 37 (5): 884 888.

15. Khan I, Bew D, Elias DA, Lewis D, Meacock LM (2014): Mechanisms of injury and CT findings in bowel and mesenteric trauma. Clin Radiol., 69 (6): 639-647.

16. Heller MT, Shah A, Furlan A (2014): MDCT of acute conditions affecting the mesenteric vasculature. Clin Radiol., 69 (7): 765-772.

17. LeBedis CA, Anderson SW, Soto JA (2012): CT imaging of blunt traumatic bowel and mesenteric injuries. Radiol Clin North Am., 50 (1): 123-136.

18. Levine CD, Gonzales RN, Wachsberg RH, Ghanekar D (1997): CT findings of bowel and mesenteric injury. J Comput Assist Tomogr., 21 (6): 974-979.

19. Hefny AF, Kunhivalappil FT, Matev N, Avila NA, Bashir MO, Abu-Zidan FM (2015): Usefulness of free intraperitoneal air detected by CT scan in diagnosing bowel perforation in blunt trauma: experience from a community-based hospital. Injury. Injury, 46(1):100-4.

20. Fang JF, Wong YC, Lin BC, Hsu YP, Chen MF (2006): Usefulness of multidetector computed tomography for the initial assessment of blunt abdominal trauma patients. World $\mathbf{J}$ Surg., 30 (2): 176-182.

21. Hines J, Rosenblat J, Duncan DR, Friedman B, Katz DS (2013): Perforation of the mesenteric small bowel: etiologies and CT findings. Emerg Radiol., 20 (2): 155-161.

22. Rodriguez C, Barone JE, Wilbanks TO, Rha CK, Miller K (2002): Isolated free fluid on computed tomographic scan in blunt abdominal trauma: a systematic review of incidence and management. J Trauma, 53 (1): 79-85.

23. Lee SS, Park SH (2013): Computed tomography evaluation of gastrointestinal bleeding and acute mesenteric ischemia. Radiol Clin North Am., 51 (1): 29-43.

24. Brody JM, Leighton DB, Murphy BL, Abbott GF, Vaccaro JP, Jagminas L, Cioffi WG (2000): CT of blunt trauma bowel and mesenteric injury: typical findings and pitfalls in diagnosis. Radiographics, 20 (6): $1525-1536$. 